\title{
The determinant of the Laplacian on the $n$-sphere
}

\author{
by
}

\author{
H. Kumagai (Fukuoka)
}

1. Introduction and statement of results. Let $\Delta$ be the Laplacian on the compact Riemann manifold $M$. Then $\Delta$ has a discrete spectrum

$$
0=\lambda_{0}<\lambda_{1} \leq \lambda_{2} \leq \ldots,
$$

for which we introduce the zeta-function

$$
Z(s)=\sum_{n=1}^{\infty} \frac{1}{\lambda_{n}^{s}}, \quad \operatorname{Re} s=\sigma>\alpha
$$

(0-energy level excluded), absolutely convergent in a half-plane in view of the Weyl law. It is shown that $Z(s)$ can be continued to the region including 0 , and we can interpret the (otherwise) divergent "determinant"

$$
\operatorname{det}^{\prime} \Delta=\prod_{n=1}^{\infty} \lambda_{n}
$$

as the zeta-regularized product (or the functional determinant)

$$
\operatorname{det} \Delta=e^{-Z^{\prime}(0)}
$$

which is the determinant of the Laplacian in the title, where we note that since

$$
Z^{\prime}(s)=-\sum_{n=1}^{\infty} \frac{\log \lambda_{n}}{\lambda_{n}^{s}}, \quad \sigma>\alpha,
$$

$e^{-Z^{\prime}(0)}$ is formally equal to the product $\operatorname{det}^{\prime} \Delta$ of positive eigenvalues.

For compact Riemann surfaces with constant curvature the determinants of the Laplacian have recently been studied extensively by D'Hoker-Phong [5], [6], Sarnak [12], Voros [16] (for non-compact case, see e.g. Efrat [7]), in view of their relevance to superstring theory. The main feature is the

1991 Mathematics Subject Classification: 11M41, 81C91.

Key words and phrases: regularized determinant, Hurwitz zeta-function, eigenvalues of the Laplacian. 
computation of determinants in terms of values of the Selberg zeta-function, where multiple gamma functions play important roles.

For compact Riemann manifolds of higher dimensions, such as the unit $n$-sphere

$$
S^{n-1}=\left\{\left(x_{1}, \ldots, x_{n}\right) \in \mathbb{R}^{n} \mid x_{1}^{2}+\ldots+x_{n}^{2}=1\right\},
$$

the regularized determinants have also been studied, notably by Weisberger [17], [18], Vardi [14] and Choi [1], [2].

They computed the determinant of the Laplacian of the unit $n$-sphere $S^{n-1}$ with standard metric in terms of the values of the derivative of the Hurwitz (Riemann) zeta-function at 0 . The unit 2-sphere case which was computed in all the above papers is of interest again due to its relationship to superstring theory (see Vardi [14], Osgood, Phillips, Sarnak [10], Weisberger [17], [18]).

Our purpose is to give a closed form evaluation of $\operatorname{det} \Delta_{n}$ for any $n$, and give a corrected version of Vardi's Theorems 1.1 and 1.2 of [14], thus compiling all existing special cases in higher dimensions.

We note that our elementary method applies to any dimension, while Weisberger's method seems to be restricted to the 2-dimensional case, and Choi's method seems too complicated to modify it to higher dimensions (even 3).

We now set out to state our theorems. We recall from [13], [15] that the eigenvalues of the standard Laplacian on the $n$-sphere are $k(k+n-1)$ with multiplicity

$$
\left(\begin{array}{c}
k+n \\
n
\end{array}\right)-\left(\begin{array}{c}
k+n-2 \\
n
\end{array}\right) \quad(k=0,1,2, \ldots) .
$$

We form the zeta-function

$$
Z(s)=Z_{n}(s)=\sum_{k=1}^{\infty} \frac{\left(\begin{array}{c}
k+n \\
n
\end{array}\right)-\left(\begin{array}{c}
k+n-2 \\
n
\end{array}\right)}{(k(k+n-1))^{s}}
$$

(zero mode excluded), which is absolutely convergent for $\operatorname{Re} s=: \sigma>n / 2$; we shall prove in Lemma 3 that it can be continued to a half-plane including the origin. Thus we can define the (regularized) determinant det $\Delta_{n}$ of the Laplacian on the $n$-sphere by

$$
\operatorname{det} \Delta_{n}=e^{-Z^{\prime}(0)} .
$$

We shall prove the following closed form of $\operatorname{det} \Delta_{n}$.

TheOREM 1. For arbitrary dimension n,

$$
\operatorname{det} \Delta_{n}=\exp \left(\sum_{d=0}^{n-1} T_{n, d} H_{n-1, d}^{\prime}(0)\right),
$$

where $H_{n-1, d}^{\prime}(0)$ and $T_{n, d}$ are as given in Lemmas 3 and 2, respectively: 


$$
\begin{aligned}
H_{n-1, d}^{\prime}(0)= & 2 \zeta^{\prime}(-d)+\sum_{l=0}^{d-1}\left(\begin{array}{l}
d \\
l
\end{array}\right)(1-n)^{d-l} \zeta^{\prime}(-l) \\
& +(-1)^{d} \sum_{l=2}^{n-1}(n-l-1)^{d} \log l \\
& -\frac{2}{d+1}\left(-\frac{n-1}{2}\right)^{d+1} \sum_{\substack{l=1 \\
2 \nmid l}}^{d}\left(\begin{array}{l}
d+1 \\
l+1
\end{array}\right) \sum_{\substack{j=1 \\
2 \nmid j}}^{l} \frac{1}{j}, \\
T_{n, d}= & \frac{1}{n !} \sum_{r=d+1}^{n} s(n, r)\left(\begin{array}{l}
r \\
d
\end{array}\right)\left(n^{r-d}-(n-2)^{r-d}\right),
\end{aligned}
$$

with $s(n, r)$ denoting the Stirling numbers of the first kind defined by the Newton expansion $[4]$

Corollary. We have

$$
(x)_{n}=\sum_{r=0}^{n} s(n, r) x^{r} .
$$

(i) $\operatorname{det} \Delta_{1}=4 \pi^{2}$,

(ii) $\operatorname{det} \Delta_{2}=A^{4} e^{1 / 6}$,

(iii) $\operatorname{det} \Delta_{3}=\pi \exp \left(\zeta(3) / \pi^{2}\right)$,

(iv) $\operatorname{det} \Delta_{4}=\frac{1}{3} e^{-(2 / 3) \zeta^{\prime}(-3)} A^{13 / 3} e^{83 / 144}$,

and similarly for higher dimensions, where A denotes the Glaisher-Kinkelin constant defined by

$\log A=\lim _{n \rightarrow \infty}\left(\log \left(1^{1} 2^{2} \ldots n^{n}\right)-\left(\frac{n^{2}}{2}+\frac{n}{2}+\frac{1}{12}\right) \log n+\frac{n^{2}}{4}\right)=-\zeta^{\prime}(-1)+\frac{1}{12}$.

From Theorem 1 we immediately deduce

THEOREM 2. For arbitrary dimension n,

(i) there are computable rational numbers $\alpha_{n}, \beta_{n}, \gamma_{n}, \tau_{n, 1}, \ldots, \tau_{n, n-1}$ with $\tau_{n, n-1}=-4 /(n-1) !, \alpha_{n} \neq 0$, such that

$$
\operatorname{det} \Delta_{n}=\alpha_{n}^{\beta_{n}} e^{\gamma_{n}} \prod_{m=0}^{n-1} e^{\tau_{n, m} \zeta^{\prime}(-m)} ;
$$

(ii) there are computable rational numbers $A_{n}, B_{n}, C_{n}, Q_{n, 1}, \ldots, Q_{n, n}$ with $Q_{n, n}=2^{n+1} /\left(2^{n}-1\right), A_{n} \neq 0$, such that

$$
\operatorname{det} \Delta_{n}=A_{n}^{B_{n}} e^{C_{n}} \prod_{m=1}^{n} \Gamma_{m}(1 / 2)^{Q_{n, m}},
$$

where $\Gamma_{n}(x)$ denotes the multiple gamma function (cf. Choi [1], Vardi [14]). 
We intentionally use the same notation as in Vardi [14], but they may have slightly different meanings.

\section{Proofs}

Lemma 1. For $\lambda=0,1,2, \ldots, \alpha>0$ and $|z|<\alpha$ we have

$$
\begin{aligned}
\sum_{m=2}^{\infty} \frac{z^{m}}{m+\lambda} \zeta(m, \alpha)= & \sum_{k=0}^{\lambda}\left(\begin{array}{l}
\lambda \\
k
\end{array}\right) \zeta^{\prime}(-k, \alpha-z) z^{-k}-\zeta^{\prime}(-\lambda, \alpha) z^{-\lambda} \\
& -\sum_{m=0}^{\lambda-1} \frac{z^{-m}}{\lambda-m} \zeta(-m, \alpha) \\
& -\frac{z}{\lambda+1}(\psi(\lambda+1)-\psi(\alpha)+\gamma),
\end{aligned}
$$

where $\psi=\Gamma^{\prime} / \Gamma$ denotes the Euler digamma function.

Lemma 2. We have the decomposition

$$
Z(s)=Z_{n}(s)=\sum_{k=1}^{\infty} \frac{\left(\begin{array}{c}
k+n \\
n
\end{array}\right)-\left(\begin{array}{c}
k+n-2 \\
n
\end{array}\right)}{(k(k+n-1))^{s}}=\sum_{d=0}^{n-1} T_{n, d} H_{n-1, d}(s),
$$

where

$$
T_{n, d}=\frac{1}{n !} \sum_{r=d+1}^{n} s(n, r)\left(\begin{array}{l}
r \\
d
\end{array}\right)\left(n^{r-d}-(n-2)^{r-d}\right),
$$

$s(n, r)$ denoting the Stirling numbers of the first kind and

$$
H_{d}(s)=H_{n, d}(s)=\sum_{k=1}^{\infty} \frac{k^{d}}{(k(k+n))^{s}} .
$$

Lemma 3 (cf. Proposition 3.1 of Vardi [14]).

$$
\begin{aligned}
H_{d}^{\prime}(0)=H_{n, d}^{\prime}(0)= & \sum_{k=1}^{n}(k-n)^{d} \log k-\frac{1}{2^{d}} \cdot \frac{(-n)^{d+1}}{d+1} \sum_{\substack{l=1 \\
2 \nmid l}}^{d}\left(\begin{array}{l}
d+1 \\
l+1
\end{array}\right) \sum_{\substack{j=1 \\
2 \nmid j}}^{l} \frac{1}{j} \\
& +\zeta^{\prime}(-d)+(-n)^{d} \sum_{r=0}^{d}\left(\begin{array}{l}
d \\
r
\end{array}\right) \frac{\zeta^{\prime}(-r)}{(-n)^{r}} .
\end{aligned}
$$

We split the proof of Lemma 3 into a few sublemmas. Lemma 4 gives a detailed decomposition of $H_{d}(s)$, which gives an analytic continuation for $\sigma>-1$ of $H_{d}(s)$ as well as a handy formula for $H_{d}^{\prime}(s)$. Then in Lemma 5 we obtain by a limit process a closed form evaluation of $H_{d}^{\prime}(0)$, and in Lemma 6 we collect auxiliary formulas that enable us to simplify the formula in Lemma 5. 
Lemma 4. For $\sigma>-1$, we have

(i) $H_{d}(s)=\sum_{l=0}^{d}\left(\begin{array}{l}d \\ l\end{array}\right)\left(-\frac{n}{2}\right)^{d-l}(\zeta(2 s-l, 1+n / 2)$

$$
\begin{aligned}
& \left.+\sum_{r=1}^{\infty} \frac{(n / 2)^{r}}{r !} \cdot \frac{\Gamma(s+r)}{\Gamma(s)} \zeta(2 s-l+r, 1+n / 2)\right) \\
& +\sum_{l=0}^{d}\left(\begin{array}{l}
d \\
l
\end{array}\right) \sum_{m=1}^{\infty}\left(-\frac{n}{2}\right)^{d-l+m} \frac{\Gamma(s+m)}{m ! \Gamma(s)} \zeta(2 s-l+m, 1+n / 2) \\
& +\sum_{l=0}^{d}\left(\begin{array}{l}
d \\
l
\end{array}\right) \sum_{m=1}^{\infty}\left(-\frac{n}{2}\right)^{d-l+m} \frac{\Gamma(s+m)}{m ! \Gamma(s)} \\
& \times \sum_{r=1}^{\infty} \frac{(n / 2)^{r}}{r !} \cdot \frac{\Gamma(s+r)}{\Gamma(s)} \zeta(2 s-l+r+m, 1+n / 2),
\end{aligned}
$$

(ii) $H_{d}^{\prime}(s)=\sum_{l=0}^{d}\left(\begin{array}{l}d \\ l\end{array}\right)\left(-\frac{n}{2}\right)^{d-l}\left(2 \zeta^{\prime}(2 s-l, 1+n / 2)\right.$

$$
\begin{aligned}
& +\sum_{r=1}^{\infty} \frac{(-n / 2)^{r}}{r !} \cdot \frac{\Gamma(s+r)}{\Gamma(s)}(\psi(s+r)-\psi(s)) \\
& \left.\times \zeta(2 s-l+r, 1+n / 2)+2 \zeta^{\prime}(2 s-l+r, 1+n / 2)\right) \\
& +\sum_{l=0}^{d}\left(\begin{array}{l}
d \\
l
\end{array}\right)\left(-\frac{n}{2}\right)^{d-l} \\
& \times \sum_{r=1}^{\infty} \frac{(-n / 2)^{r}}{r !} \cdot \frac{\Gamma(s+r)}{\Gamma(s)}((\psi(s+r)-\psi(s)) \\
& \left.\times \zeta(2 s-l+r, 1+n / 2)+2 \zeta^{\prime}(2 s-l+r, 1+n / 2)\right) \\
& +\sum_{l=0}^{d}\left(\begin{array}{l}
d \\
l
\end{array}\right)\left(-\frac{n}{2}\right)^{d-l} \sum_{m=1}^{\infty} \frac{(-n / 2)^{m}}{m !} \cdot \frac{\Gamma(s+m)}{\Gamma(s)}(\psi(s+m)-\psi(s)) \\
& \times \sum_{r=1}^{\infty} \frac{(n / 2)^{r}}{r !} \cdot \frac{\Gamma(s+r)}{\Gamma(s)} \zeta(2 s-l+r+m, 1+n / 2) \\
& +\sum_{l=0}^{d}\left(\begin{array}{l}
d \\
l
\end{array}\right)\left(-\frac{n}{2}\right)^{d-l} \sum_{m=1}^{\infty} \frac{(-n / 2)^{m}}{m !} \cdot \frac{\Gamma(s+m)}{\Gamma(s)} \\
& \times \sum_{r=1}^{\infty} \frac{(n / 2)^{r}}{r !} \cdot \frac{\Gamma(s+r)}{\Gamma(s)}((\psi(s+r)-\psi(s)) \\
& \left.\times \zeta(2 s-l+r+m, 1+n / 2)+2 \zeta^{\prime}(2 s-l+r+m, 1+n / 2)\right) .
\end{aligned}
$$


Pr o of. To prove (i), applying the binomial theorem, we obtain

$$
H_{d}(s)=\sum_{l=0}^{d}\left(\begin{array}{l}
d \\
l
\end{array}\right)\left(-\frac{n}{2}\right)^{d-l} G_{l}(s),
$$

where

(4)

$$
\begin{aligned}
& G_{l}(s) \\
= & \sum_{r=0}^{\infty} \frac{\Gamma(s+r)}{r ! \Gamma(s)}\left(\frac{n}{2}\right)^{r} \frac{1}{(k+n / 2)^{r}} \sum_{m=0}^{\infty} \frac{\Gamma(s+m)}{m ! \Gamma(s)}\left(-\frac{n}{2}\right)^{m} \frac{1}{(k+n / 2)^{m}} .
\end{aligned}
$$

Substituting this in (3) and changing the order of summation, we deduce that

$$
\begin{aligned}
H_{d}(s)= & \sum_{l=0}^{d}\left(\begin{array}{l}
d \\
l
\end{array}\right) \sum_{m=0}^{\infty}\left(-\frac{n}{2}\right)^{d-l+m} \frac{\Gamma(s+m)}{m ! \Gamma(s)} \\
& \times \sum_{r=0}^{\infty} \frac{(n / 2)^{r}}{r !} \cdot \frac{\Gamma(s+r)}{\Gamma(s)} \zeta(2 s-l+r+m, 1+n / 2),
\end{aligned}
$$

the process being legitimate by absolute convergence.

For $\sigma>-1$, we use the estimate

$$
\zeta(2 s-l+r+m, 1+n / 2) \ll(1+n / 2)^{-2 \sigma+l-r-m}
$$

for $2 \sigma-l+r+m>1$ to conclude that the sums over $m$ and $r$ in (5) are absolutely convergent for $\sigma>-1$, thus giving an analytic continuation of $H_{d}(s)$.

The formula (i) follows by dividing the sums suitably.

The formula (ii) follows directly from (i) by applying Leibniz's rule, thereby completing the proof.

LEMMA 5. We have

$$
H_{d}^{\prime}(0)=\sum^{+}+\sum^{-}+\sum_{l=0}^{d}\left(\begin{array}{l}
d \\
l
\end{array}\right)\left(-\frac{n}{2}\right)^{d-l} \frac{1}{2}\left(\frac{n}{2}\right)^{l+1} \sum_{m=1}^{l} \frac{(-1)^{m}}{m(l+1-m)},
$$

where

$$
\begin{aligned}
\sum^{+}= & \sum_{l=0}^{d}\left(\begin{array}{l}
d \\
l
\end{array}\right)\left(-\frac{n}{2}\right)^{d-l}\left(2 \zeta^{\prime}(-l, 1+n / 2)\right. \\
& \left.+\sum_{\substack{r=1 \\
r \neq l+1}}^{\infty} \frac{(n / 2)^{r}}{r} \zeta(r-l, 1+n / 2)+\frac{(n / 2)^{l+1}}{l+1} \cdot \frac{1}{2} \sum_{j=1}^{l} \frac{1}{j}\right)
\end{aligned}
$$

and 


$$
\begin{aligned}
\sum^{-}= & \sum_{l=0}^{d}\left(\begin{array}{l}
d \\
l
\end{array}\right)\left(-\frac{n}{2}\right)^{d-l}\left(\sum_{\substack{m=1 \\
m \neq l+1}}^{\infty} \frac{(-n / 2)^{m}}{m} \zeta(m-l, 1+n / 2)\right. \\
& \left.+\frac{(-n / 2)^{l+1}}{l+1} \cdot \frac{1}{2} \sum_{j=1}^{l} \frac{1}{j}\right) .
\end{aligned}
$$

LEMMA 6. We have the evaluations

$$
\sum_{l=0}^{d}\left(\begin{array}{l}
d \\
l
\end{array}\right)\left(-\frac{n}{2}\right)^{d-l} \sum_{m=0}^{l}\left(\begin{array}{c}
l \\
m
\end{array}\right) \zeta^{\prime}(-m)\left(\frac{n}{2}\right)^{l-m}=\zeta^{\prime}(-d),
$$

(ii) $\quad \sum_{l=0}^{d}\left(\begin{array}{l}d \\ l\end{array}\right)\left(-\frac{n}{2}\right)^{d-l} \sum_{m=0}^{l}\left(\begin{array}{l}l \\ m\end{array}\right) \zeta^{\prime}(-m, 1+n)\left(-\frac{n}{2}\right)^{l-m}$

$$
=\sum_{m=0}^{d}\left(\begin{array}{c}
d \\
m
\end{array}\right)(-n)^{d-m} \zeta^{\prime}(-m, 1+n) .
$$

Proof of Lemma 3. Substituting from Lemma 1, we see that

$$
\begin{aligned}
\sum^{+}+\sum^{-}= & \sum_{l=0}^{d}\left(\begin{array}{l}
d \\
l
\end{array}\right)\left(-\frac{n}{2}\right)^{d-l}\left(-\frac{1}{2} \cdot \frac{(n / 2)^{l+1}+(-n / 2)^{l+1}}{l+1} \sum_{j=1}^{l} \frac{1}{j}\right) \\
& +\sum_{l=0}^{d}\left(\begin{array}{l}
d \\
l
\end{array}\right)\left(-\frac{n}{2}\right)^{d-l} \sum_{m=0}^{l}\left(\begin{array}{c}
l \\
m
\end{array}\right) \zeta^{\prime}(-m)\left(\frac{n}{2}\right)^{l-m} \\
& +\sum_{l=0}^{d}\left(\begin{array}{l}
d \\
l
\end{array}\right)\left(-\frac{n}{2}\right)^{d-l} \sum_{m=0}^{l}\left(\begin{array}{c}
l \\
m
\end{array}\right) \zeta^{\prime}(-m, 1+n)\left(\frac{n}{2}\right)^{l-m}
\end{aligned}
$$

and

$$
\begin{aligned}
H_{d}^{\prime}(0)= & \zeta^{\prime}(-d)+\sum_{m=0}^{d}\left(\begin{array}{c}
d \\
m
\end{array}\right)(-n)^{d-m} \zeta^{\prime}(-m, 1+n) \\
& -\frac{2}{d+1}\left(-\frac{n}{2}\right)^{d+1} \sum_{\substack{l=1 \\
2 \nmid l}}^{d}\left(\begin{array}{l}
d+1 \\
l+1
\end{array}\right) \sum_{\substack{j=1 \\
2 \nmid j}}^{l} \frac{1}{j}
\end{aligned}
$$

Using Lemma 6, and expressing

$$
\zeta^{\prime}(-m, 1+n)=\zeta^{\prime}(-m)+\sum_{r=2}^{n} r^{m} \log r
$$

completes the proof.

Proof of Theorem 2. (i) is a restatement of Theorem 1 and is a corrected form of Theorem 1.4 of Vardi [14], while (ii) follows from (i) and Theorem 1.1 of Vardi [14], and is a corrected form of Theorem 1.3 of Vardi [14]. 


\section{Remarks}

REMARK 1 (The unit circle $S^{0}$ ). The determinant $\operatorname{det} \Delta_{1}$ of $S^{0}$ with standard Laplacian $\Delta_{1}=d^{2} / d x^{2}$ is

$$
\operatorname{det} \Delta_{1}=(2 \pi)^{2} \text {. }
$$

Remark 2 (The unit disc $S^{1}$ ). Vardi's formula for $F_{2}^{\prime}(0)$ in Proposition 4.4 of [14] is correct. As a matter of fact, the proof of Theorem 1.4 (p. 505) gives the incorrect value of $F_{2}^{\prime}(0)$ :

$$
F_{2}^{\prime}(0)=4 \zeta^{\prime}(-1)+\zeta^{\prime}(0)-1 / 2,
$$

but in the statement of Proposition 4.4, the author omitted $\zeta^{\prime}(0)$ by mistake to give a correct value. Accordingly, the formulas containing $F_{2}^{\prime}(0)$ would have been as follows. The second formula in Proposition 4.5 would read

$$
e^{\zeta^{\prime}(-1)}=\left(\operatorname{det} \Delta_{2}\right)^{-1 / 4} e^{1 / 8}
$$

the formula for $\Gamma_{2}(1 / 2)$ in Theorem 1.1 would read

$$
\Gamma_{2}(1 / 2)=\left(\operatorname{det} \Delta_{2}\right)^{3 / 8}\left(\operatorname{det} \Delta_{1}\right)^{1 / 8} 2^{-11 / 16} e^{-1 / 16} \pi^{-3 / 16}
$$

and that in Theorem 1.2 would read

$$
\operatorname{det} \Delta_{2}=\Gamma_{2}(1 / 2)^{8 / 3} 2^{1 / 2}(e / \pi)^{1 / 16} .
$$

The formulas for $\operatorname{det} \Delta_{2}$ as given in Vardi (except for the one in Theorem 1.2 in which the factor $\pi^{-2 / 3}$ is missing) are in conformity with the formulas of Weisberger [17], [18] and of Choi [1], [2] and Quine and Choi [11]. The error comes from the incorrect argument in Proposition 3.1 which lacks the evaluation of the infinite series $B(0)$, which looks rather difficult. In the statement of Proposition 3.1 this term $B(0)$ is missing, but the value given there is very close to the correct one.

Choi's argument in the case $n=2$ (see [1]) follows exactly Voros' and gives the correct value of $\operatorname{det} \Delta_{2}$. His statement on p. 166 of [1] is rather misleading because he says there that his value coincides with that of Vardi. However, Choi's correct value does not coincide with (6), but rather with the value given in Theorem 1 (Corollary (ii)).

For general metrics, see e.g. [9].

REMARK 3 (The unit sphere). The only correct existing formula is Choi's main theorem [1], [2] and Quine and Choi [11]. Choi's method uses the shifted generating Dirichlet series process of Voros', which requires a considerable amount of calculation with sophisticated multiple gamma function, and it looks rather hopeless to go on further to higher dimensions with Voros' method. Actually, the proof occupies the main body of Choi's thesis.

Vardi's general closed formulas in his Theorems 1.1 and 1.2 are wrong. Choi's remark in [1] was again rather misleading in that he calculates the same value (unknown in the literature) in two ways using both his results 
and Vardi's results, and concludes that they give different values. This leaves a possibility that both might be wrong, but this defect was rescued in [8], and Choi's result for det $\Delta_{3}$ is correct and coincides with ours.

Since our closed formula (Corollary) gives correct values for both det $\Delta_{2}$ and $\operatorname{det} \Delta_{3}$, it is of considerable trust.

REMARK 4 (Higher dimensions). After presenting our results at the Japan-Korea Number Theory Conference, Dec. 24-27, 1997 held at Saga University, we learned about the paper of Quine and Choi [11], which gives a closed formula for det $\Delta_{n}$ for any $n$. Their method avoids the computation of the infinite series involving the Hurwitz zeta-function by an ingenious trick of introducing a regularization lemma (Lemma 1), in which cancellation of terms in our Lemma 5 is effected by showing $G^{\prime}(0)=0$, and the proof is subtler than ours. We believe, however, that our method has its own right, clarifying how those cancel one another.

Acknowledgments. The author would like to express his deepest gratitude to Prof. S. Kanemitsu for suggesting the problem and constant encouragement and to Mr. Yoshimoto for his invaluable help in writing the paper. The author also wishes to thank Prof. J. S. Choi for informing about his joint paper with Quine [11]. Finally, the author would like to thank Prof. S. Egami wholeheartedly for suggesting a method for continuing analytically the zeta-function, which enabled him to evaluate special values thereof.

The Editors of Acta Arithmetica have kindly drawn our attention to the paper by Choi and Srivastava [3] which treats the 3-dimensional Laplacian $\Delta_{3}$ using the double gamma-function, and give the same result as ours in this case. The author would like to thank the Editors for this remark.

\section{References}

[1] J. S. Choi, Ph.D. thesis, Florida State Univ., 1991.

[2] - Determinant of Laplacian on $S^{3}$, Math. Japon. 40 (1994), 155-166.

[3] J. S. Choi and H. M. Srivastava, An application of the theory of the double Gamma function, Kyushu J. Math. 53 (1999), 209-222.

[4] L. Comtet, Advanced Combinatorics, Reidel, 1974.

[5] E. D'Hoker and D. H. Phong, Multiloop amplitudes for the bosonic Polyakov string, Nuclear Phys. B 269 (1986), 205-234.

[6] - - - On determinants of Laplacians on Riemann surfaces, Comm. Math. Phys. 104 (1986), 537-545.

[7] I. Efrat, Determinants of Laplacians on surfaces of finite volume, ibid. 119 (1988), 443-451; erratum, ibid. 138 (1991), 607.

[8] S. Kanemitsu, M. Katsurada and M. Yoshimoto, On the Hurwitz-Lerch zeta function, to appear. 
[9] E. Onofri, On the positivity of the effective action in a theory of random surface, Comm. Math. Phys. 86 (1982), 321-326.

[10] B. Osgood, R. Phillips and P. Sarnak, Extremals of determinants of Laplacians, J. Funct. Anal. 80 (1988), 148-211.

[11] J. R. Quine and J. Choi, Zeta regularized products and functional determinants on spheres, Rocky Mountain J. Math. 26 (1996), 719-729.

[12] P. Sarnak, Determinants of Laplacians, Comm. Math. Phys. 110 (1987), 113-120.

[13] A. Terras, Harmonic Analysis on Symmetric Space and Applications. I, Springer, 1985.

[14] I. Vardi, Determinants of Laplacians and multiple gamma functions, SIAM J. Math. Anal. 19 (1988), 493-507.

[15] N. Yu. Vilenkin, Special Functions and the Theory of Group Representations, Amer. Math. Soc. Transl. 22, Amer. Math. Soc., 1968, p. 494.

[16] A. Voros, Spectral functions, special functions and the Selberg zeta function, Comm. Math. Phys. 110 (1987), 439-465.

[17] W. I. Weisberger, Conformal invariants for determinants of Laplacians on Riemann surfaces, ibid. 112 (1987), 633-638.

[18] - Normalization of the path integral measure and the coupling constants for the bosonic strings, Nuclear Phys. B 284 (1987), 171-200.

Department of Electrical Engineering

University of Kinki, Iizuka

Fukuoka 820-8555, Japan

E-mail: de9801hk@ed.fuk.kindai.ac.jp 